\title{
Detection of Neospora caninum from Farm-Bred Young Blue Foxes (Alopex lagopus) in China
}

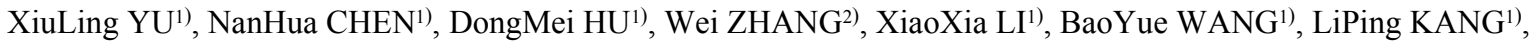 \\ XiangDong $\mathrm{LI}^{3)}$, Qun $\mathrm{LIU}^{2)}$ and KeGong TIAN ${ }^{1) *}$ \\ ${ }^{1)}$ National Veterinary Diagnostic Center, Ministry of Agriculture, Beijing 100094, ${ }^{2)}$ Parasitology Laboratory, Department of Veterinary \\ Preventive Medicine, College of Veterinary Medicine, China Agricultural University, Beijing 100094 and \\ ${ }^{3}$ Key Laboratory of Animal Parasitology, Ministry of Agriculture, Department of Veterinary Public Health, Shanghai Veterinary Research \\ Institute, Chinese Academy of Agricultural Sciences, Shanghai 200232, PR China
}

(Received 14 April 2008/Accepted 16 September 2008)

ABSTRACT. Neospora caninum has been detected in several wild mammalian species, i.e., deers, coyotes, dingoes, and foxes. Farm-bred foxes were rarely reported to be affected by the parasite. In this study, we detected for the first time the infection of N.caninum in the farm-bred young blue foxes (Alopex lagopus) in China. Neospora-like tissue cysts were observed in brains and kidneys of the foxes by histopathological and immunohistochemical examinations. One hundred and three sera from the clinically normal vixens were tested for the presence of N.caninum and Toxoplasma gondii antibodies by two commercial ELISA test kits. Twenty-eight of 103 (27.2\%) sera were positive for $N$. caninum and 1 serum $(0.97 \%)$ was positive for $T$. gondii. A portion of the Nc5 gene of $N$. caninum was amplified from the DNA extracted from the fox brains by semi-nested PCR, further confirmed the existence of $N$. caninum among the farm-bred fox herd in China.

KEY WORDS: Alopex lagopus, Neospora caninum, seroprevalence.

J. Vet. Med. Sci. 71(1): 113-115, 2009

Neospora caninum is an obligate intracellular protozoan parasite with a worldwide distribution [9]. Since its first recognition in dogs in Norway and the description of a new genus Neospora and species Neospora caninum [3, 6], neosporosis has emerged as a serious disease causing fatal neuromuscular disease in dogs and abortion or stillbirth in cattles and other mammals of veterinary importance [2]. The full life-cycle of this parasite has not yet been elucidated. Tachyzoites, tissue cysts, and oocysts are considered to be the three infectious stages in its life-cycle [5]. Blue foxes (Alopex lagopus) are members of the Canidae family which are widely bred in China. Previous epidemiological studies have indicated that foxes have been exposed to $N$. caninum, and they can serve as intermediate hosts as well as definitive hosts $[1,11]$, though attempts to induce oocyst production have been unsuccessful [10]. Seroepidemiological studies have indicated that $N$. caninum were prevailing among red foxes in several countries [7]. However, meager information is available on the prevalence of $N$. caninum among foxes in China. In this study, serological, histopathologic, immunohistochemical and molecular evidence are presented, which implicate $N$. caninum for the first time in China as the main aetiological agent causing paralysis and death in farm-bred young blue foxes.

The foxes were bred in a farm located in Hebei province, China. The farm had more than 200 newborn foxes in 2007 , the morbility of the group was more than $60 \%$, and the mortality of the affected foxes exceeded $50 \%$. After excluding

\footnotetext{
* Correspondence to: Tian, K., National Veterinary Diagnostic Center, Ministry of Agriculture No. 2, Yuanmingyuan West Road, Haidian District, Beijing, 100094, P. R. China. e-mail: tiankg@263.net
}

the infection of a variety of pathogens such as pathogenic bacteria (e.g. E. coli, Staphylococcus and Salmonella) or viruses (e.g. fox distemper virus, parvovirus and encephalitis virus), a total of 103 fox sera were collected to test for antibody of $N$. caninum (c-ELISA VMRD Laboratories, Pullman, Washington, U.S.A.) and T. gondii (Anti-Toxoplasma IgG Antibody ELISA Kit, ZhuHai S.E.Z. HaiTai Biological Pharmaceuticals, Ltd, China) using commercial ELISA kits according to the manufacturer's recommendations.

Necropsy of blue foxes were performed according to the guidelines of the Public Health Service Policy on Humane Care and Use of Laboratory Animals. Samples of brain, heart, lung, liver, spleen, kidney and lymph node were fixed in $10 \%$ neutral buffered formalin at necropsy and processed routinely for histological examination. Fixed tissues were embedded in paraffin, sectioned, stained with haematoxylin and eosin (HE), Periodic Acid Schiff (PAS) and Giemsa, respectively. Immunohistochemistry was employed to study the above of formalin-fixed paraffin-embedded specimens. The primary antibodies were canine antisera against $N$. caninum and mouse antisera against $T$. gondii ( kindly provided by Dr. Xuenan Xuan from Japan). N. caninumnegative canine sera were used to test non-specific background staining.

DNA was extracted from brain tissues of the young blue foxes. The genomic Nc5 region was selected as the target sequence for DNA amplification by semi-nested PCR using the $N$. caninum-specific primer pair $\mathrm{Np} 6 / 21$, followed by amplification with secondary primers Np6/7 [12].

Of the 103 sera tested, twenty-eight reacted with $N$. caninum. Antibody to T. gondii was only found in one of serum 


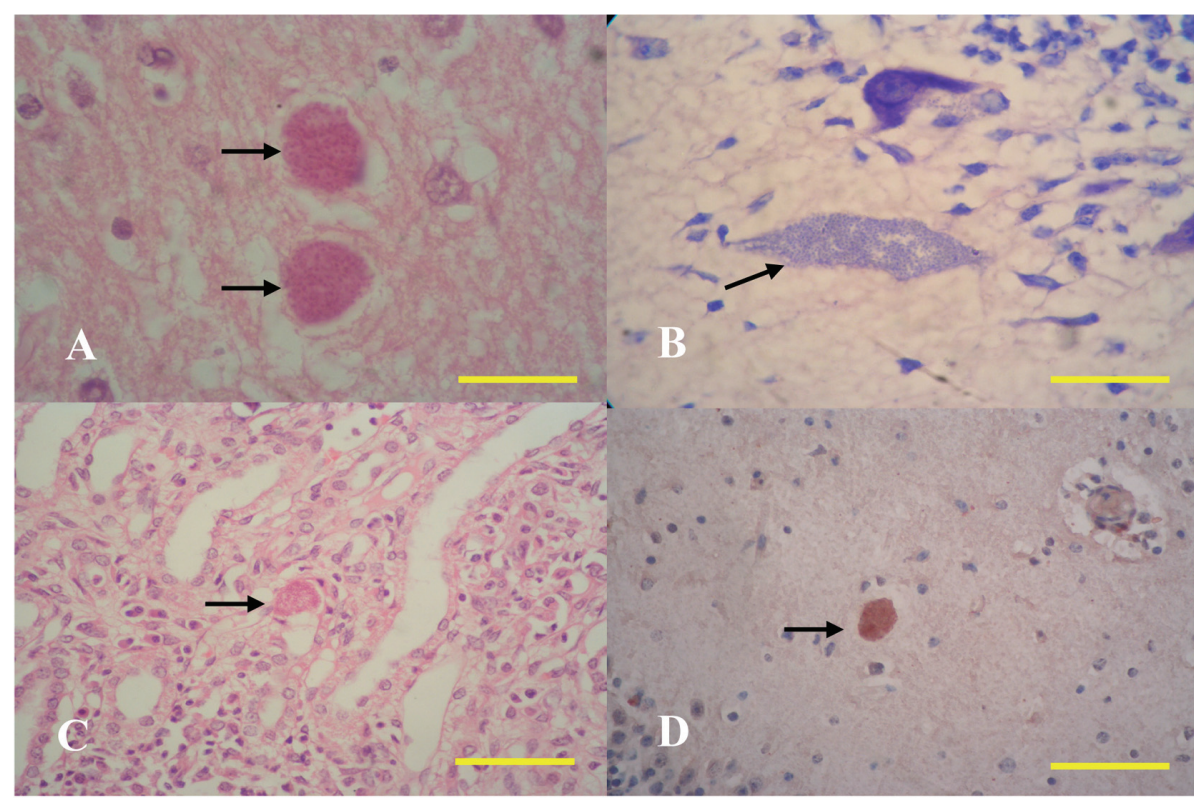

Fig. 1. Photomicrographs of formalin-fixed paraffin-embeded tissue sections. (A) N. caninum cysts in the cerebrum. HE stain. (B) A clump of tachyzoites of $N$. caninum around granular layer of the cerebellum. Giemsa stain. (C) N. caninum cyst in the renal tubules. PAS stain. (D) Tissue cyst in the cerebrum stained positively with the canine anti- $N$. caninum antisera. Scale bar in A and $\mathrm{B}=20 \mu \mathrm{m}$; scale bar in $\mathrm{C}$ and $\mathrm{D}=50 \mu \mathrm{m}$.

samples, which was negative reaction for $N$. caninum. Seropositive five foxes with $N$. caninum were small in size with clinical signs of coarse fur, inappetence, emaciation, fever, and particularly neurologic signs including depression, ataxia and paralysis. At necropsy, small foci of infarcts on the edge of the spleen were observed. There were visible foci of necrosis and rugosity in the surface of the kidney. The boundary between medulla and cortex was unclear. Cerebral pia mater was coarse, and hydrocephalus was visible in all five foxes.

Markedly congestion, small foci of necrosis were visible in the sections of the cerebrum, midbrain, cerebellum and spinal cord. Multifocal nonsuppurative encephalitis was observed, which consisted of perivascular cuffings of colloid cells. Many Neospora-like tissue cysts, round in shape and $25-100 \mu \mathrm{m}$ in diameter with walls up to $3 \mu \mathrm{m}$ thick, were found around the inflammatory foci in the cerebrum (Fig. 1A), and medulla oblongata. A cluster of tachyzoites were also found in the brain tissue (Fig. 1B). Immunohistochemically, tissue cysts in the cerebrum stained positively with the canine anti- $N$. caninum antisera (Fig. 1D), but showed no significant reaction to the mouse anti- $T$. gondii antisera and $N$. caninum-negative canine sera which exclude the possibility of non-specific background staining.

Following amplification of DNA from five foxes using $N$. caninum seminested PCR, 227bp products were observed (Fig. 2) from the positive control and four of five foxes (No. 2 sample was negative). Two PCRs using primers specific for $N$. caninum failed to yield an amplification products from negative control ( $T$. gondii DNA and water). DNA sequencing of gel-cleaned PCR products and alignment of Nc5 sequences revealed $97-99 \%$ similarity to the Nc5 sequence of $N$. caninum (data not shown), thus providing a definitive genomic identification of the infection.

PCR plays an important role in the diagnosis of $N$. caninum infection. Specific partial Nc5 sequence of $N$. caninum was amplified by PCR from positive control and DNA of four foxes, PCR for T. gondii was negative. Since we observed Neospora-like tissue cysts or tachyzoites in all the five fox brains, the omit of detection by PCR may due to non-uniform distribution or very low number of parasites in tissues [4].

According to the previous documents, tissue cysts are more likely to form in the central nervous system rather than other tissues [6]. To our surprise, $N$. caninum tissue cysts have also been found in the renal tubules ( 2 of 5 foxes) with PAS staining (Fig. 1C). The thickness of the cyst wall in the tissues was the same as previously reported [8]. No cysts were observed in other tissues.

Recently, the first identification of $N$. caninum infection in aborted bovine foetus in China was reported [13], yet there was no report on $N$. caninum infection in foxes. The results of our study verified for the first time the prevalence of $N$. caninum in china. More fox sera need to be collected from other regions to delineate the significance of $N$. caninum as the main aetiological agent causing paralysis and death in farm-bred young blue foxes in China. This work is now carrying on in our laboratory.

ACKNOWLEDGEMENTS. The authors are very grateful 


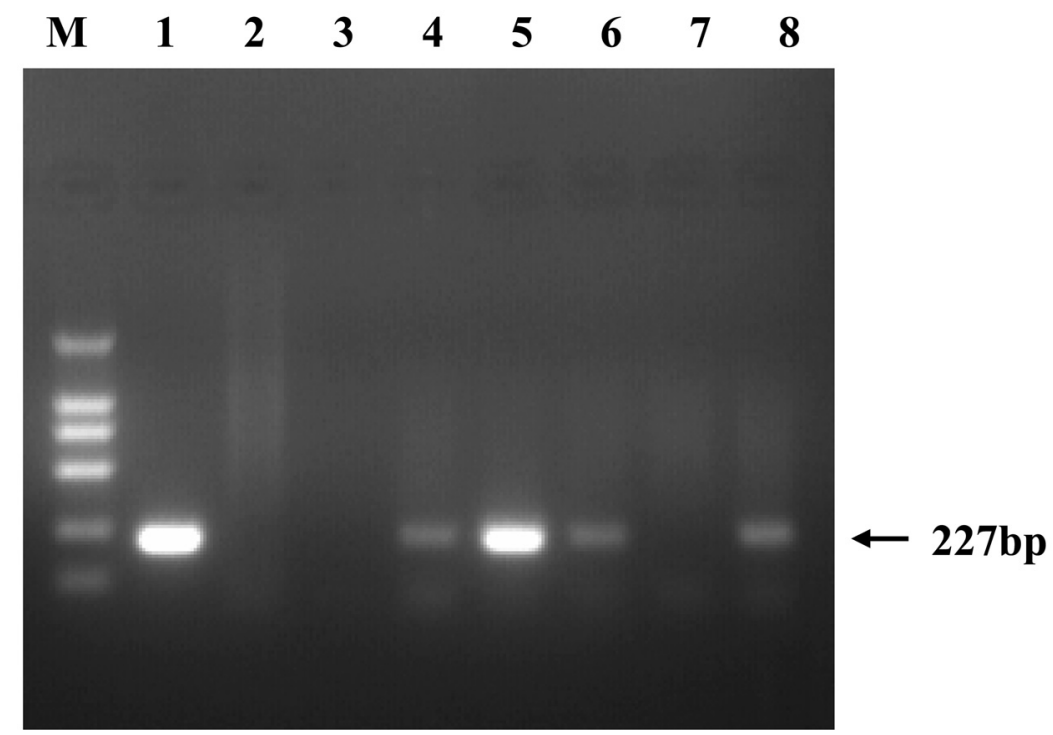

Fig. 2. Semi-nested PCR for $N$. caninum using Np6/Np7 primers. M=2 kb DNA ladder; Lane 1, Positive control (DNA of the Nc-1 isolate of N. caninum); Lane 2, Negative control (DNA of $T$. gondii); Lane 3, Negative control (Water); Lanes 4-8, DNA extracted from the fox brains, we note the specific amplification products of $227 \mathrm{bp}$ in positive control and lanes 4, 5, 6, 8 .

to Dr. Xuenan Xuan (National Research Center for Protozoan Diseases, Obihiro University, Japan) for providing the canine anti- $N$. caninum antisera, mouse anti- $T$. gondii antisera and DNA of the Nc-1 isolate of $N$. caninum used as positive control in semi-nested PCR. We gratefully acknowledge the technical assistance of personnel in National Veterinary Diagnostic Center, Ministry of Agriculture, PR China.

\section{REFERENCES}

1. Almería, S., Ferrer, D., Pabón, M., Castellá, J. and Mañas, S. 2002. Red foxes (Vulpes vulpes) are a natural intermediate host of Neospora caninum. Vet. Parasitol. 107: 87-294.

2. Barber, J. S., Gasser, R.B., Ellis, J., Reichel, M.P., McMillan, D. and Trees, A.J. 1997. Prevalence of antibodies to Neospora caninum in different canid populations. J. Parasitol. 83: 10561058.

3. Bjerkås, I., Mohn, S.F. and Presthus, J. 1984. Unidentified cyst-forming sporozoon causing encephalomyelitis and myositis in dogs. Zeitschrift Fur Parasitenkund. 70: 271-274.

4. De, Marez, T., Liddell, T.S., Dubey, J.P., Jenkins, M.C. and Gasbarre, L. 1999. Oral infection of calves with Neospora caninum oocysts from dogs: humoral and cellular immune responses. Int. J. Parasitol. 29: 1647-1657.

5. Dubey, J.P. 2003. Review of Neospora caninum and neosporosis in animals. Korean J. Parasitol. 41: 1-16.

6. Dubey, J.P., Carpenter, J.L., Speer, C.A., Topper, M.J. and Uqqla, A. 1988. Newly recognized fatal protozoan disease of dogs. Am. Vet. Med. Assoc. 92: 1269-1285.
7. Hamilton, C.M., Gray, R., Wright, S.E., Grangadharan, B., Laurenson, K. and Innes, E.A. 2005. Prevalence of antibodies to Toxoplasma gondii and Neospora caninum in red foxes (Vulpes vulpes) from around the UK. Vet. Parasitol. 130: 169173.

8. Jardine, J.E. 1996. The ultrastructure of bradyzoites and tissue cysts of Neospora caninum in dogs: absence of distinguishing morphological features between parasites of canine and bovine origin. Vet. Parasitol. 62: 231-240.

9. McAllister, M., Wills, R.A., McGuire, A.M., Jolley, W.R., Tranas, J.D., Williams, E.S., Lindsay, D.S., Björkman, C. and Belden, E.L. 1999. Ingestion of Neospora caninum tissue cysts by Mustela species. Int. J. Parasitol. 29: 1531-1536.

10. Schares, G., Heydorn, A.O., Cüppers, A., Mehlhorn, H., Geue, L., Peters, M. and Conraths, F.J. 2002. In contrast to dogs, red foxes (Vulpes vulpes) did not shed Neospora caninum upon feeding of intermediate host tissues. Parasitol. Res. 88: 44-52.

11. Wapenaar, W., Barkema, H.W., Schares, G., Rouvinen-Watt, K., Zeijlemaker, L., Poorter, B., O'Handley, M.R., Kwok, O.C.H. and Dubey, J.P. 2006. Evaluation of four serological techniques to determine the seroprevalence of Neospora caninum in foxes (Vulpes vulpes) and coyotes (Canis latrans) on Prince Edward Island, Canada. Vet. Parasitol. 145: 51-58.

12. Yamage, M., Flechtner, O. and Gottstein, B. 1996. Neospora caninum: specific oligonucleotide primers for the detection of brain "cyst" DNA of experimentally infected nude mice by the polymerase chain reaction (PCR). J. Parasitol. 82: 272-279.

13 Zhang, W., C. Deng, Q. Liu, J. Liu, M. Wang, K.G. Tian, X.L. $\mathrm{Yu}$, and D.M. Hu. 2007. First identification of Neospora caninum infection in aborted bovine foetuses in China. Vet. Parasitol. 149: 72-76. 